\title{
Irritable bowel syndrome treatment: cognitive behavioral therapy versus medical treatment
}

Majid Mahvi-Shirazi ${ }^{1}$, Ali Fathi-Ashtiani' ${ }^{2}$ Sayed-Kazem Rasoolzade-Tabatabaei ${ }^{3}$, Mohsen Amini $^{2}$

1Department of Psychology, Islamic Azad University, Buinzahra Branch, Iran 2Behavioral Sciences Research Center, Department of Psychology, Baqiyatallah University of Medical Sciences, Tehran, Iran

${ }^{3}$ Department of Psychology, Tarbiat Modares University, Tehran, Iran

Submitted: 11 June 2008

Accepted: 24 August 2008

Arch Med Sci 2012; 8, 1: 123-129

DOI: 10.5114 /aoms.2012.27292

Copyright (c) 2012 Termedia \& Banach

\section{Abstract}

Introduction: The study aims to investigate two kinds of treatment in patients suffering from irritable bowel syndrome (IBS) and consequently compares its efficacy on improving the symptoms and mental health of patients; one with just medical treatment and another through a combination of psychotherapy and medical treatment.

Material and methods: Applying general sampling, 50 IBS patients were selected from among those who used to refer to a Gastroenterology Clinic. After physical and mental evaluations based on ROME-II scale and SCL-90-R questionnaires, the subjects were randomly superseded into: the control group with medical treatment and, the case group with a combination of medical and psychological treatments. The acquired data were then analyzed through $t$-test and Mann-Whitney U-test.

Results: The findings show that the mental health of patients receiving cognitive behavioral therapy along with the medical treatment was higher than those of the control group at post-test level. It was observed that the therapy reduces the disability caused by IBS. Comparatively, while the cognitive therapy and medical treatments cured $80 \%$ of the patients, those receiving cognitive therapy alone showed an extensive reduction of symptoms.

Conclusions: Considering the role of cognitive behavioral therapy, it is therefore recommend that such patients be managed by a combined team of gastroenterologists and psychologists.

Key words: irritable bowel syndrome, cognitive behavioral therapy, medical treatment, psychological status.

\section{Introduction}

Irritable bowel syndrome or IBS is a functional disorder of the gastrointestinal system and clinically it can be diagnosed with the symptoms of changes in bowel movement, pain or bloating. The IBS is prevalent among about $10 \%$ to $20 \%$ of the general population and as the most widespread disorder which is associated with psycho-social factors as compared to other gastrointestinal disorders [1]. So far as the treatment of IBS gastroenterologists usually prescribe uniform medications, the most important of them being:

- Lopramide, which minimizes diarrhea by reducing muscles contraction and fluids discharge in intestine.

\author{
Corresponding author: \\ Prof. Ali Fathi-Ashtiani \\ Behavioral Sciences Research \\ Centere \\ Department of Psychology \\ Baqiyatallah University \\ of Medical Sciences \\ Tehran, Iran \\ E-mail: afa1337@gmail.com
}


- Lomotil (Diphenoxylate) is used to minimize diarrhea in the IBS patients.

- Dimethicon (Malokus), Alfagalactizeoas and Charcoal to some extent reduce bloating and stretching.

- Matoclopramide is one of the muscle flexing medicines (Prokinetics) that with the increasing activities of intestine could help in relieving constipation and bloating.

- Amitriptyline or Nortriptyline can help in reducing stomachache during sleep. These medicines have much effect especially in the IBS disease for overcoming diarrhea.

With regard to IBS treatment, studies show that a combination of medical treatment and psychotherapy, especially cognitive behavioural therapy, has a significant response in decreasing the symptoms. As a matter of fact, the cognitive behavioural therapy-based treatments have accompanied new challenges in the field of IBS treatment [2]. Furthermore, a quick review of the literature reveals the necessity of defining the psychological aspect of this syndrome. Some studies show that cognitive behavioural therapy (CBT) along with medical treatment leads to different results when compared with medical treatment alone [3-6]. The findings of a crosssectional study showed that IBS is accompanied by high psychological distress and patients who have always applied self-treatment encounter severe psychological dysfunction [7]. One study shows that IBS needs a multi-component approach including medical treatment, diet and psychotherapy [8]. Another study reveals that psychotherapy, especially cognitive behavioural therapy, hypnotherapy and psychoanalysis, is effective in treating IBS patients [5]. In the course of a similar study in Australia, 7 IBS patients received 8 sessions of cognitive behavioural therapy. Before initiating the treatment, all these patients were assessed for psychological performance and severity of GI signs. After the treatment, it was observed that five of them did not have any IBS signs. Although the frequency of expression of symptoms by patients did not decrease, the frequency of depression and anxiety decreased, significantly. As a whole, the results indicated that cognitive behavioural therapy reduces the disability caused by IBS; however, it did not affect the expression of the symptoms by patients [9].

A study conducted at the Center of Stress and Anxiety of an American university reviewed all psychological treatments applied to persons suffering from IBS and found that hypnotherapy, cognitive therapy and short-term psychoanalysis were significantly efficient [3].

Boyce [5] presents his findings this way: With reference to the biopsychosocial model of interaction between gut function and emotion in IBS, psychological therapies are intended to break the neg- ative feedback loop between emotion and gut function in order to reduce symptoms. Attention must be paid to a number of elements in the psychological approaches including a detailed assessment, psycho-education, support, and reassurance.

Edward et al. [4] reviewed the efficacy of cognitive therapy and hypnotherapy. It seems that they were quite effective compared to placebo and their effects also lasted for nearly 4 years. Furthermore, the close cooperation between the psychotherapist and the gastroenterologist has been the most effective in controlling the symptoms. Taylor compared patients suffering from IBS who had marked concealed aggressiveness with those who had mild concealed aggressiveness and concluded that the first group had a poorer prognosis when they received medical treatment alone (without psychotherapy) [9].

Based on previous studies, the current study aims to compare the efficacy of medical and cognitive behavioural treatments in improving the mental health level of IBS patients. And thus, the study intends to answer the question whether cognitive behavioural therapy along with pharmaceutical treatment is more effective than that of medical treatment alone, in improving symptoms and mental health of IBS patients.

\section{Material and methods}

This is a kind of quasi-experimental study, which has been conducted on all IBS patients referred to a GI clinic. With reference to inclusion criteria for this study (an age group between 18 and 60 years, subjects without any history of mental or psychological diseases), 50 of them were selected through the assessment by a gastroenterologist and filling in the ROME-II questionnaire and then they were divided into the case and control groups. Both groups filled in SCL-90-R and demographic questionnaires under the supervision of a psychologist. To collect data, in addition to a researcher-made demographic questionnaire (age, sex, occupation, education, disease type, history of treatment, beginning of treatment and severity of symptoms), the following tools were applied.

\section{ROME-II questionnaire (IBS symptoms index)}

Based on clinical diagnostic criteria, the questionnaire was prepared with the help of a gastroenterologist and includes questions related to the presence or the absence of IBS symptoms. A high score shows the severity of the disease. This questionnaire contains 14 yes/no questions, where a patient gets one point for each of the positive responses approved by a gastroenterologist. The rest of the questions are multiple choice and patients score one point if they choose a particu- 
lar choice related to IBS. Finally, the collection of scores determines the severity of the disease. The higher the score is, the more probable the diagnosis and the severity of the IBS are. For instance, in question 1 (Are you evaluating the patient for IBS?), if the response is positive, the patient receives one point. Or in question 4 (Is there any change in the appearance or the consistency of the stool?), if the gastroenterologist approves it, the patient receives one point. It is noteworthy that the ROME-II questionnaire must be filled in by a gastroenterologist during the examination.

\section{SCL-90-R questionnaire}

This questionnaire contains 90 questions, which aim to evaluate psychological symptoms in 9 different aspects: Somatization, Obsessive-Compulsive, Interpersonal Sensitivity, Depression, Anxiety, Hostility, Phobic Anxiety, Paranoid Ideation and Psychoticism. Scoring and interpretation of the questionnaire are obtained on the basis of three co-efficient indices: the general symptom index (GSI), discomfort index and collective symptoms. The first draft of this questionnaire was introduced by Dragoits, Lithman and Cuvey in 1973 and was put to revision in 1983. The credit and admissibility of this questionnaire were reported as 0.72 to 0.90 and 0.36 to 0.73 , respectively. Furthermore, sensitivity, specificity and efficiency of this test have also been reported, with high validity as 0.94, 0.98 and 0.96 , respectively.

\section{Therapy method}

Cognitive behavioural therapy (CBT) was conducted for the case group during 8 consecutive weekly sessions in two months. Along with the psychotherapy sessions, IBS patients took the following conventional medications as prescribed by gastroenterologists:

- Loperamide was prescribed in order to reduce muscle contraction and fluid discharge in bowel/intestine as well as to minimize diarrhoea;

- Lomotil (diphenoxylate) was prescribed to minimize diarrhoea in the IBS patients;
- Simticon (Malokus), Alfagalactizeoas and charcoal to some extent reduce bloating and stretching;

- Metoclopramide is one of the muscle flexing medicines (Broachenitic) that with the increasing activities of intestine can help in relieving constipation and bloating;

- Amitriptyline or nortriptyline can help in reducing stomach ache during sleep. These medicines have many effects especially in IBS disease for overcoming diarrhoea.

The control group was placed under medical treatment alone by the same gastroenterologist. They also received medicines similar to the one given to the case group during the course of treatment. Thereafter, the two groups were also compared for reduction of IBS syndromes as well as regards the level of mental health.

In this way, at the end, all patients in both the groups were re-evaluated by the same gastroenterologist using the ROME-II questionnaire. Similarly, the SCL-90-R questionnaire was also re-executed in both the case and the control groups. The results acquired from these two questionnaires were compared in two stages of pre-test and posttest. To analyse the data, $t$-test and Mann-Whitney $U$ test were applied.

\section{Results}

Demographic data showed $42 \%, 36 \%$ and $22 \%$ of symptoms in the age groups of 17-27, 28-38 and $39-48$ years, respectively. The majority of patients (40\%) had a high school diploma, and 30\% had an associate diploma. Forty percent of the subjects were self-employed while $22 \%$ were students.

To answer the question whether CBT combined with medical treatment is more effective than medical treatment alone in managing IBS patients, initially the scores of the subjects of the two groups were calculated on the ROME-II scale at pre-test and post-test stages. Then, the mean and the standard deviation were calculated and mean differences were tested using the independent $t$-test (Table I).

As Table I shows, the differences between mean scores of each group in ROME-II were not signifi-

Table I. $t$ Test results for ROME-II scores in both groups at pre-test and post-test and their differences

\begin{tabular}{|c|c|c|c|c|c|c|c|c|}
\hline \multirow[t]{2}{*}{ Stages } & \multirow[t]{2}{*}{ Group } & \multirow[t]{2}{*}{ Mean } & \multirow[t]{2}{*}{ SD } & \multicolumn{2}{|c|}{ Lewin test } & \multirow[t]{2}{*}{ Value of $t$} & \multirow[t]{2}{*}{$\mathrm{d} f$} & \multirow[t]{2}{*}{ Value of $p$} \\
\hline & & & & F ratio & $p$ & & & \\
\hline \multirow[t]{2}{*}{ Pre-test } & Case & 10.85 & 2.13 & 1.238 & 0.271 & 0.089 & 48 & 0.930 \\
\hline & Control & 10.80 & 1.78 & & & & & \\
\hline \multirow[t]{2}{*}{ Post-test } & Case & 4.00 & 1.83 & 2.527 & 0.118 & 6.900 & 48 & 0.001 \\
\hline & Control & 8.10 & 2.24 & & & & & \\
\hline \multirow[t]{2}{*}{ Pre-test and post-test } & Case & 6.85 & 0.30 & 1.672 & 0.202 & 6.860 & 48 & 0.001 \\
\hline & Control & 2.70 & -0.46 & & & & & \\
\hline
\end{tabular}


Table II. Comparison of statistical indices of SCL-90-R test between case and control groups at pre-test

\begin{tabular}{|c|c|c|c|c|c|c|}
\hline SCL-90-R scales & Group & Mean & SD & Value of $t$ & Degrees of freedom & Value of $p$ \\
\hline \multirow[t]{2}{*}{ Somatization } & Case & 1.62 & 0.98 & 0.149 & 48 & 0.88 \\
\hline & Control & 1.65 & 0.78 & & & \\
\hline \multirow[t]{2}{*}{ Obsessive-compulsive } & Case & 1.74 & 0.80 & 0.022 & 48 & 0.98 \\
\hline & Control & 1.74 & 0.79 & & & \\
\hline \multirow[t]{2}{*}{ Interpersonal sensitivity } & Case & 1.57 & 0.73 & 0.849 & 48 & 0.40 \\
\hline & Control & 1.38 & 0.72 & & & \\
\hline \multirow[t]{2}{*}{ Depression } & Case & 2.01 & 0.64 & 1.820 & 48 & 0.07 \\
\hline & Control & 1.56 & 0.98 & & & \\
\hline \multirow[t]{2}{*}{ Anxiety } & Case & 1.59 & 0.96 & 0.210 & 48 & 0.83 \\
\hline & Control & 1.53 & 0.92 & & & \\
\hline \multirow[t]{2}{*}{ Hostility } & Case & 1.55 & 0.83 & 0.170 & 48 & 0.86 \\
\hline & Control & 1.60 & 1.09 & & & \\
\hline \multirow[t]{2}{*}{ Phobic anxiety } & Case & 0.97 & 0.70 & 0.290 & 48 & 0.77 \\
\hline & Control & 0.91 & 0.79 & & & \\
\hline \multirow[t]{2}{*}{ Paranoid ideation } & Case & 1.86 & 0.81 & 1.510 & 48 & 0.13 \\
\hline & Control & 1.48 & 0.91 & & & \\
\hline \multirow[t]{2}{*}{ Psychoticism } & Case & 1.40 & 0.69 & 1.300 & 48 & 0.19 \\
\hline & Control & 1.16 & 0.58 & & & \\
\hline \multirow[t]{2}{*}{ GSI } & Case & 1.59 & 0.57 & 0.760 & 48 & 0.44 \\
\hline & Control & 1.45 & 0.69 & & & \\
\hline
\end{tabular}

Table III. Comparison of statistical indices of SCL-90-R test between case and control groups at post-test

\begin{tabular}{|c|c|c|c|c|c|c|}
\hline SCL-90-R scales & Group & Mean & SD & Value of $t$ & Degrees of freedom & Value of $p$ \\
\hline \multirow[t]{2}{*}{ Somatization } & Case & 0.84 & 0.63 & 4.90 & 48 & 0.0001 \\
\hline & Control & 1.90 & 0.81 & & & \\
\hline \multirow[t]{2}{*}{ Obsessive-compulsive } & Case & 1.06 & 0.49 & 4.34 & 48 & 0.0001 \\
\hline & Control & 1.96 & 0.83 & & & \\
\hline \multirow[t]{2}{*}{ Interpersonal sensitivity } & Case & 0.86 & 0.52 & 4.04 & 48 & 0.0001 \\
\hline & Control & 1.68 & 0.80 & & & \\
\hline \multirow[t]{2}{*}{ Depression } & Case & 1.01 & 0.53 & 3.23 & 48 & 0.0020 \\
\hline & Control & 1.80 & 0.99 & & & \\
\hline \multirow[t]{2}{*}{ Anxiety } & Case & 0.64 & 0.45 & 4.57 & 48 & 0.0001 \\
\hline & Control & 1.69 & 0.94 & & & \\
\hline \multirow[t]{2}{*}{ Hostility } & Case & 0.80 & 0.64 & 3.93 & 48 & 0.0001 \\
\hline & Control & 1.88 & 1.10 & & & \\
\hline \multirow[t]{2}{*}{ Phobic anxiety } & Case & 0.40 & 0.41 & 3.94 & 48 & 0.0001 \\
\hline & Control & 1.25 & 0.89 & & & \\
\hline \multirow[t]{2}{*}{ Paranoid ideation } & Case & 1.00 & 0.69 & 3.10 & 48 & 0.0030 \\
\hline & Control & 1.79 & 0.97 & & & \\
\hline \multirow[t]{2}{*}{ Psychoticism } & Case & 0.82 & 0.40 & 3.56 & 48 & 0.0010 \\
\hline & Control & 1.41 & 0.66 & & & \\
\hline \multirow[t]{2}{*}{ GSI } & Case & 0.83 & 0.38 & 4.85 & 48 & 0.0001 \\
\hline & Control & 1.71 & 0.74 & & & \\
\hline
\end{tabular}


cant at the pre-test stage. However, at post-test, this difference was significant $(p<0.001)$, i.e. the group receiving $\mathrm{CBT}$ combined with medical treatment gained lower scores at post-test of the ROME-II scale. In addition, the mean difference of subjects calculated at pre-test and post-test was significant $(p<0.001)$ for both the groups. This shows that CBT combined with medical treatment is more effective than medical treatment alone in managing IBS patients.

To test the question whether cognitive behavioural therapy along with medical treatment improves the mental health of IBS patients more effectively than medical treatment alone, first the scores of the subjects in both the groups were calculated at pre-test and post-test stages in SCL-90-R sub-scales, the general symptom index (GSI), the mean score and the standard deviation (since GSI is the mean of the mean scores of subjects on the SCL-90-R scales, it was considered the mental health index of subjects). Then, the independent $t$-test for mean scores of both groups in pre-test and Mann-Whitney $U$-test for post-test were utilized to determine the significance of mean differences of the proposed test. (With reference to significant differences between variances on some scales, the Mann-Whitney U-test was used to reconfirm and compare the results in post-test.) The results are shown in Tables II-V.

As Tables II-V show, the differences in mean scores of each group on all sub-scales and GSI in SCL-90-R at the pre-test level were not significant, but they were significant at the post-test level. Similarly, there was a significant difference between the GSI scores of pre-test and post-test $(p<0.001)$ levels. This means the mental health of patients receiving $\mathrm{CBT}$ along with the medical treatment was higher than that of the control group at the posttest level.

\section{Discussion}

The present study indicates that the CBT combined with the medical treatment was more effective than the medical treatment alone in reducing IBS symptoms. The control group, which only received medical treatment, showed a reduction of symptoms but the level was not significant. In other words, patients receiving combination therapies (CBT plus medical treatment) gained lower scores in ROME-II clinical indices. This shows that IBS symp-

Table IV. Results of statistical indices of Mann-Whitney $U$ test for comparing GSI difference between case and control groups at post-test

\begin{tabular}{|c|c|c|c|}
\hline Subscale & Group & $X$ & $\mathrm{~S}$ \\
\hline \multirow[t]{2}{*}{ Somatization } & Case & 15.48 & 309.50 \\
\hline & Control & 32.18 & 965.50 \\
\hline \multirow[t]{2}{*}{ Obsessive-compulsive } & Case & 16.23 & 324.50 \\
\hline & Control & 31.68 & 950.50 \\
\hline \multirow[t]{2}{*}{ Interpersonal sensitivity } & Case & 16.33 & 326.50 \\
\hline & Control & 31.62 & 948.50 \\
\hline \multirow[t]{2}{*}{ Depression } & Case & 18.13 & 362.50 \\
\hline & Control & 30.42 & 912.50 \\
\hline \multirow[t]{2}{*}{ Anxiety } & Case & 15.50 & 310.00 \\
\hline & Control & 32.17 & 965.00 \\
\hline \multirow[t]{2}{*}{ Hostility } & Case & 16.60 & 332.00 \\
\hline & Control & 31.43 & 943.00 \\
\hline \multirow[t]{2}{*}{ Phobic anxiety } & Case & 16.98 & 339.50 \\
\hline & Control & 31.18 & 935.50 \\
\hline \multirow[t]{2}{*}{ Paranoid ideation } & Case & 18.05 & 361.00 \\
\hline & Control & 30.47 & 914.00 \\
\hline \multirow[t]{2}{*}{ Psychoticism } & Case & 17.55 & 351.00 \\
\hline & Control & 30.80 & 924.00 \\
\hline \multirow[t]{2}{*}{ GSI } & Case & 14.95 & 299.00 \\
\hline & Control & 32.53 & 976.00 \\
\hline
\end{tabular}

Table V. Results of Mann-Whitney $U$ test for comparing GSI difference between case and control groups at post-test

\begin{tabular}{|c|c|c|c|}
\hline SCL-90-R subscales & Mann-Whitney $U$ & Value of $Z$ & Value of $p$ \\
\hline Somatization & 99.500 & -3.974 & 0.0001 \\
\hline Obsessive-compulsive & 114.500 & -3.679 & 0.0001 \\
\hline Interpersonal sensitivity & 116.500 & -3.640 & 0.0001 \\
\hline Depression & 152.500 & -2.924 & 0.0030 \\
\hline Anxiety & 100.000 & -3.970 & 0.0001 \\
\hline Hostility & 122.000 & -3.537 & 0.0001 \\
\hline Phobic anxiety & 129.500 & -3.391 & 0.0010 \\
\hline Paranoid ideation & 151.000 & -2.957 & 0.0030 \\
\hline Psychoticism & 141.000 & -3.157 & 0.0020 \\
\hline GSI & 89.000 & -4.179 & 0.0001 \\
\hline
\end{tabular}


toms were reduced in these patients. The case group improved in ROME-Il clinical indices compared not only to the control group but also to themselves at the pre-test level. The reduction of scores in the case group is in conformity with several studies [7, 11-15] which conclude that cognitive therapy, psychotherapy, short-term psychoanalysis and cognitive behavioural therapy have been the most effective treatments for IBS. In comparison, while the cognitive and medical treatment cured $80 \%$ of IBS patients, those receiving cognitive therapy alone showed extensive reduction of symptoms. In addition, the advantages of cognitive therapy remain for a longer time after the treatment [16]. Pinto et al. found that recognition and treatment of anxiety and depression in the subgroups of IBS patients with psychotropic drugs and cognitive therapy, for gaining more positive coping skills, may require special attention in the management of IBS [12]. It is noteworthy that when IBS patients receive combined treatment, their symptoms seem to decrease significantly. As such, the study conducted by Guthrie et al. shows that psychotherapy in IBS patients reduced stomach ache and diarrhoea, but did not affect constipation. At the same time, cognitive therapy has been proved to be useful for controlling IBS symptoms [17]. Also, Blanchard et al. stated that progressive muscle relaxation training for patients as well as training for reducing tension and relaxing could reduce IBS symptoms. When these methods are used for a longer period of over 4 years, some $50 \%$ of symptoms such as pain, nausea and bloating will be cured [18]. These studies also showed that some IBS symptoms are affected by psychotherapy more than others. In the present study, patients' scores decreased relatively after the psychotherapy. However, the ROME-II questions have not been analysed here one by one; hence, it cannot be stated which symptoms were reduced more or which ones did not change. This study is in contrast with some parts of the studies conducted by Boyce and Gilcharist, who believed that CBT reduces IBS-related distress and disabilities, but does not reduce intestinal symptoms. This shows that CBT probably increases organ sensitivity through changing the cognitive response [6].

The current study has shown that in the case group different IBS symptoms were reduced when under medical treatment (when medicines were being administered to IBS patients) combined with cognitive behavioural therapy, compared to medical treatment alone, with the reason these two methods are performed through experimental methods. As such, it seems that the two methods of CBT and medical treatment show a better cure, especially in IBS treatment, when combined together. In other words, together, these two methods are complete treatment.
Furthermore, the results also show that the mental health level of subjects suffering from IBS improved consequently under CBT combined with medical treatment than those under medical treatment only. With regards to the results of t-test and Mann-Whitney $U$-test after CBT, the case group enjoyed a higher health score than the control group. Mean scores showed that the mental health of the control group, who received only medical treatment, did not change, i.e. mean differences were not statistically significant; however, the case group enjoyed a better health score than the control group. The case group under CBT had a higher health score at the post-test level than at the pretest. These results are in line with some previous studies [5, 6]. According to Boyce et al. [6], because of the biopsychosocial model of interaction between gut function and emotion in IBS, psychological therapies are intended to break the negative feedback loop between emotion and gut function, in order to reduce symptoms. According to them, attention must be paid to a number of elements in the psychological approaches including a detailed assessment, psycho-education, support, and reassurance. The results of cognitive therapy and hypnotherapy have been reviewed several times and thus it seems they were quite effective compared to placebo and their effect lasted for nearly 4 years [4]. In one of his studies, Taylor compared patients suffering from IBS with marked concealed aggressiveness with those with mild concealed aggressiveness and concluded that the first group had a poorer prognosis when they received the medical treatment alone (without psychoterapy) [13]. Boyce and Gilcharist also showed that patients' depression and anxiety significantly decrease with the treatment [6]. Overall it can be said that the increase in mental health of IBS patients was due to the effect of the cognitive behavioural therapy combined with the medical treatment. It is therefore necessary that patients follow psychologists' advice all the time. Also, they have to take medication prescribed by gastroenterologists, as well. This is the only way one can be hopeful for a desired level of patients' health.

In conclusion, to sum up, cognitive behavioural therapy combined with medical treatment can reduce IBS symptoms and improve patients' mental health. Therefore, it is necessary that patients be referred to psychologists for psychotherapy while they are under medical treatment.

\section{References}

1. Häuser W, Grandt D. Measuring quality of life in gastroenterology - concepts, instruments and problems [German]. Z Gastroentero 2001; 39: 475-81.

2. Heymann-Mönnikes I, Arnold R, Florin I, Herda C, Melfsen S, Mönnikes H. The combination of medical treatment 
plus multicomponent behavioral therapy is superior to medical treatment alone in the therapy of irritable bowel syndrome. Am J Gastroenterology 2000; 95: 981-94.

3. Edward B, Bharucha L. Psychological aspects of assessment and treatment of IBS, stress and anxiety disorder. Centre of an American University, USA 2001; 358-367.

4. Edward B, Louis K. Psychological treatment in IBS patient, best practice and research. Clinl Gastroenterol 1999; 13: 473-87.

5. Boyce P. Psychologic therapies for irritable bowel syndrome. Curr Treat Options Gastroenterol 2001; 4: 323-31.

6. Boyce P, Gilchrist J, Talley NJ, Rose D. Cognitive-behaviour therapy as a treatment for irritable bowel syndrome: a pilot study. Aust N Z J Psychiatry 2000; 34: 300-9.

7. Zndedel N, Nsani M. IBS and psychological disorders in university students. Sci J Iranian Assoc Gastroenterol Hematol 2006; 11: 22-6.

8. Winery BRM. Combination therapy in IBS. J Consult Clin Psychology 2002; 40: 725-38.

9. Blanchard EB, Keefer L, Galovski TE, Taylor AE, Turner SM. Gender differences in psychological distress among patients with irritable bowel syndrome. J Psychosom Res 2001; 50: 271-5.

10. Wilson GT. Behavior research and therapy the problem of structural indeterminacy in multidimensional symptom report instruments. The case of SCL-90-R. 1999;37(7): 685-701.

11. Latimer P. Psychophysiologic disorders: a critical appraisal of concept and theory illustrated with reference to the irritable bowel syndrome (IBS). Psychol Med 1979; 9: 71-8.

12. Pinto C, Lele MV, Joglekar AS, Panwar VS, Dhavale HS. Stressful life-events, anxiety, depression and coping in patients of irritable bowel syndrome. J Assoc Physicians India 2000; 48: 589-93.

13. Tanum L, Malt UF. Personality traits predict treatment outcome with an antidepressant in patients with functional gastrointestinal disorder. Scand J Gastroenterol 2000; 35: 935-41.

14. Lydiard RB. Irritable bowel syndrome, anxiety and depression: what are the links? J Clin Psychiatry 2001; 62 (Suppl 8): 38-45.

15. Solmaz M, Kavuk I, Sayar K. Psychological factors in the irritable bowel syndrome. Eur J Med Res 2003; 8: 549-56.

16. Payne A, Blanchard EB. A controlled comparison of cognitive therapy and self-help support groups in the treatment of irritable bowel syndrome. J Consult Clin Psychol 1995; 63: 779-86.

17. Guthrie E, Creed F, Dawson D, Tomenson B. A randomized controlled trial of psychotherapy in patients with refractory irritable bowel syndrome. Br J Psychiatry 1993; 163: 315-21.

18. Blanchard EB, Schwarz SP. Adaptation of a multicomponent treatment for irritable bowel syndrome to a smallgroup format. Biofeedback Self Regul 1987; 12: 63-9. 